\title{
Factores asociados con el nivel de conocimientos y la actitud hacia la investigación en estudiantes de medicina en Perú, 2011
}

\author{
John A. Cabrera-Enríquez, ${ }^{1}$ César Cruzado-Mendoza, ${ }^{2}$ \\ Nelson Purizaca-Rosillo, ${ }^{3}$ Rubén O. López-Samanamú, ${ }^{4}$ \\ Yasmín Lajo-Aurazo, ${ }^{2}$ Eric R. Peña-Sánchez, ${ }^{5}$ \\ Moisés Apolaya-Segura ${ }^{6}$ y Cristian Díaz-Vélez 7
}

Forma de citar Cabrera-Enríquez JA, Cruzado-Mendoza C, Purizaca-Rosillo N, López-Samanamú RO, Lajo-Aurazo Y, Peña-Sánchez ER, et al. Factores asociados con el nivel de conocimientos y la actitud hacia la investigación en estudiantes de medicina en Perú, 2011. Rev Panam Salud Publica. 2013;33(3):166-73.

RESUMEN Objetivo. Identificar los factores asociados con el nivel de conocimientos y la actitud relacionados con la investigación en los estudiantes de pregrado de la carrera de medicina en Perú. Métodos. Estudio transversal analítico. Se aplicó un cuestionario validado sobre conocimientos, actitud y prácticas relacionados con la investigación a una muestra representativa de estudiantes de 17 facultades de medicina de Perú. Se realizó el análisis simple y bifactorial para las variables categóricas y cuantitativas, con ajuste de la correlación intraclase. Se modelaron las razones de prevalencia mediante análisis bifactorial y multifactorial con modelos lineales generalizados.

Resultados. Se analizaron los cuestionarios de 1554 estudiantes (51,0\% mujeres) con una edad promedio de 20,5 años $\pm 2,86$. Solo $46,7 \%$ de los encuestados tenía un buen nivel de conocimientos y $37,7 \%$ una adecuada actitud hacia la investigación. Las variables asociadas con el buen nivel de conocimientos fueron: el año académico del encuestado a partir del $4 .^{\circ}$, pertenecer a un grupo de investigación y contar con un asesor. Tener una adecuada actitud hacia la investigación se asoció con ser mujer y tener un asesor. No se encontraron diferencias entre el nivel de conocimientos y la actitud de los estudiantes de las universidades estudiadas. Conclusiones. En general, el nivel de conocimientos sobre la investigación es deficiente y la actitud hacia ella, inadecuada. Para cambiar esta situación, es prioritario hacer cambios curriculares que garanticen la incorporación de los estudiantes a la investigación desde el inicio de la carrera y desarrollar competencias en investigación en los últimos años de estudio.

Palabras clave Educación de pregrado en medicina; política de investigación en salud; promoción de la investigación; Perú.

1 Sociedad Científica de Estudiantes de Medicina, Universidad Nacional Pedro Ruiz Gallo, Chiclayo, Lambayeque, Perú. La correspondencia se debe dirigir a John A. Cabrera-Enríquez. Correo electrónico: jace3715@gmail.com

2 Sociedad Científica de Estudiantes de Medicina, Universidad de San Martín de Porres, Lima, Perú.
Sociedad Científica de Estudiantes de Medicina, Universidad Nacional de Piura, Piura, Perú.

4 Sociedad Científica Huachana de Estudiantes de Medicina, Universidad Nacional José Faustino Sánchez Carrión, Huacho, Lima, Perú.

5 Gerencia Regional de Salud, Lambayeque, Perú.

6 Dirección de Sanidad de la Fuerza Aérea del Perú, Lima, Perú.
La investigación se define como un proceso intencional de construcción de nuevos conocimientos que permite in-

Oficina de Inteligencia Sanitaria, Hospital Nacional Almanzor Aguinaga Asenjo, Lambayeque, Perú. 
terpretar los fenómenos del medio, en interacción con sujetos que también se transforman en beneficio de la sociedad (1). Al ayudar a identificar y explicar las diversas falencias de una sociedad, la investigación contribuye a conocer más cabalmente la realidad social de cada país $(1,2)$. Así, la investigación en salud es una herramienta imprescindible para la generación de conocimientos y experiencias útiles que inciden directamente en la solución de los problemas sanitarios (3) y conducen a la toma de decisiones mejor fundamentadas, en todos los niveles de gestión, para garantizar una mejor calidad de vida.

En Perú, la actividad científica es insuficiente. Si se toma como indicador el número de publicaciones científicas en relación con la población del país (4), la producción científica generada en Perú $(0,21$ artículos publicados por millón de habitantes en el año 2007) es inferior a la de otros países de América Latina, como Chile (2,14 publicaciones científicas por millón de habitantes ese mismo año), y está muy por debajo de los países líderes en la ciencia, como los Estados Unidos de América (12,86 por millón de habitantes ese mismo año).

Frente a esta realidad, en Perú se vienen desarrollando algunas estrategias dirigidas a impulsar la investigación científica desde el pregrado universitario. En 2009, se creó el Sistema Nacional de Evaluación, Acreditación y Certificación de la Calidad Educativa (SINEACE) y se elaboró el Modelo de Calidad para la Acreditación de las Carreras Profesionales Universitarias, que constituyen el pilar fundamental para la evaluación de la formación profesional (5). Entre sus objetivos está promover la investigación en pregrado - con un enfoque humanístico y dirigida a la solución de los principales problemas del país (6) - , de manera que se convierta en el principal motor de cambio y la base del progreso y la continuidad de la universidad (7). Esto significa que el estudiante debe reconocer que la etapa universitaria va mucho más allá de la mera obtención de un título profesional y que debe contribuir al cumplimiento de la función bajo la cual fue concebida la universidad: la generación de nuevos conocimientos (8).

Para los estudiantes de medicina, la investigación está estrechamente ligada a su formación y a su actividad futura (9-10), una idea ya aceptada por la mayoría de los educandos (6-7) y de la cual ganan conciencia a medida que pasan los años de carrera (11). En Perú, se encontró que $53,7 \%$ de los estudiantes de pregrado de medicina tenía buenos conocimientos sobre la investigación y 71,9\% mostró una actitud adecuada hacia esta actividad (12). No obstante, no se han establecido cuáles son las variables que pueden contribuir a fomentar la investigación, algo que permitiría repensar los programas curriculares de las facultades de medicina y otras ciencias de la salud. Esta información se hace más necesaria si se toma en cuenta el proceso de fortalecimiento del sistema de salud que está en marcha en Perú, que sigue la tendencia mundial de fomentar investigaciones que aborden las necesidades de salud, equidad y desarrollo de la población.

A raíz de este proceso, se han identificado prioridades de investigación para cada región de Perú, por lo que la formación de médicos con sólidas bases en investigación desde el pregrado debe descentralizarse también a todas las zonas del país.

El objetivo del presente estudio fue identificar los factores asociados con el nivel de conocimientos sobre la investigación y la actitud hacia ella en los estudiantes de pregrado de la carrera de medicina en Perú.

\section{MATERIALES Y MÉTODOS}

Se realizó un estudio trasversal analítico entre febrero y junio de 2011, en el que participaron estudiantes de 17 facultades de medicina de Perú, que representan $56,6 \%$ de las 30 facultades de este tipo existentes en el país.

\section{Muestra de estudio}

Se calculó que con una muestra de 1460 estudiantes encuestados se lograría un nivel de confianza de $95 \%$ y una precisión absoluta de 3,5\%, a partir de una proporción esperada de estudiantes con buen conocimiento de 53,7\% (12), un efecto de diseño de 2 y una tasa de rechazo de $10 \%$.

La selección de la muestra se realizó en dos etapas: primero se hizo un muestreo por conglomerados a partir de la lista total de alumnos por cada facultad y año académico, y luego se realizó un muestreo aleatorio simple a partir de las listas de alumnos por aula; en ambos casos se utilizó el programa Epidat 3.1.
Se incluyeron estudiantes de $1 .^{\circ}$ a $6 .^{\circ}$ años matriculados efectivamente en alguna de las 29 facultades de medicina del país que cuentan con secciones afiliadas a la Sociedad Científica Médico Estudiantil Peruana (SOCIMEP). La participación fue voluntaria.

Se consideró que el nivel de conocimientos era bueno cuando al menos $60 \%$ de las preguntas $(\geq 8)$ se respondieron correctamente y una actitud adecuada cuando se alcanzaba una puntuación $\geq 60 \%$ (al menos 77 puntos de un máximo de 110).

La recolección de datos estuvo a cargo de integrantes de la filial de la SOCIMEP de cada facultad, previamente informados del objetivo de la investigación y entrenados para atender las dudas que se presentaran. Una vez aplicados los cuestionarios a los estudiantes, se eliminaron los que no tenían los datos generales imprescindibles para el análisis (tales como universidad, edad, año de estudios y sexo) o con más de 30\% de las preguntas sin responder. En ese momento también se colectó la información propia de cada facultad (10 ítems).

Las universidades que participaron en el estudio y la muestra seleccionada en cada una de ellas se presentan en el cuadro 1.

\section{Instrumentos de recolección de datos}

Se utilizó el cuestionario sobre conocimientos, actitudes y prácticas relacionadas con la investigación, creado por Díaz-Vélez y colaboradores (12), que recoge información general personal (10 ítems), conocimientos de los estudiantes respecto a la investigación en su facultad (11 ítems) y su actitud con respecto a la investigación (22 ítems). Las respuestas se expresaron en una escala de Likert de 1 (desacuerdo total) a 5 (acuerdo total).

Este cuestionario posee elevada consistencia interna para los conocimientos y la actitud (coeficiente alfa de Cronbach de 0,82 y 0,78 , respectivamente), buena correlación intraclase de 0,81 y 0,76, respectivamente, y adecuada esfericidad (prueba de Barlett, $P<0,05$ ); el índice Kaiser-Meyer-Olkin (KMO) fue de 0,86. La varianza total explicada estableció que el cuestionario tiene una capacidad predictiva de $61 \%$.

Cinco expertos en el área de investigación (docentes e investigadores) realizaron algunas modificaciones al cuestionario para mejorar la comprensión de cada 
CUADRO 1. Universidades participantes en el estudio y muestras correspondientes, Perú, 2011

\begin{tabular}{llrr}
\hline \multicolumn{1}{c}{ Universidad } & \multicolumn{1}{c}{$\begin{array}{c}\text { Ciudad, } \\
\text { departamento }\end{array}$} & $\begin{array}{c}\text { Número de } \\
\text { encuestados }\end{array}$ & $\begin{array}{c}\text { Porcentaje } \\
\text { del total }\end{array}$ \\
\hline Universidad Católica Santa María & Arequipa, Arequipa & 123 & 7,9 \\
Universidad Nacional de Cajamarca & Cajamarca, Cajamarca & 47 & 3,0 \\
Universidad de Chiclayo & Chiclayo, Lambayeque & 54 & 3,5 \\
Universidad Nacional Pedro Ruiz Gallo & Chiclayo, Lambayeque & 34 & 2,2 \\
Universidad Nacional San Antonio Abad & Cusco, Cusco & 58 & 3,7 \\
Universidad Nacional José Faustino Sánchez Carrión & Huacho, Lima & 43 & 2,8 \\
Universidad Nacional San Luis Gonzaga & Ica, lca & 70 & 4,5 \\
Universidad Nacional de la Amazonía Peruana & Iquitos, Loreto & 35 & 2,3 \\
Universidad Privada San Martín de Porres & Lima, Lima & 309 & 19,9 \\
Universidad Privada de Ciencias Aplicadas & Lima, Lima & 79 & 5,1 \\
Universidad Ricardo Palma & Lima, Lima & 193 & 12,4 \\
Universidad Nacional de Piura & Piura, Piura & 32 & 2,1 \\
Universidad Cesar Vallejo & Piura, Piura & 21 & 1,3 \\
Universidad Nacional de Ucayali & Pucalpa, Ucayali & 38 & 2,4 \\
Universidad Nacional del Altiplano & Puno, Puno & 3,2 \\
Universidad Privada de Tacna & Tacna, Tacna & 50 & 3,2 \\
Universidad Privada Antenor Orrego & Trujillo, La Libertad & 50 & 20,5 \\
Total & & 318 & 100,0 \\
\hline
\end{tabular}

pregunta de conocimientos y actitudes y de las preguntas para identificar los factores asociados, después de lo cual se validó en una muestra de 120 estudiantes

de una de las unidades. Como durante la validación no se observaron dificultades, estos cuestionarios se incorporaron a la muestra final analizada. Se comprobó

FIGURA 1. Representación esquemática del proceso de selección de los participantes en el estudio

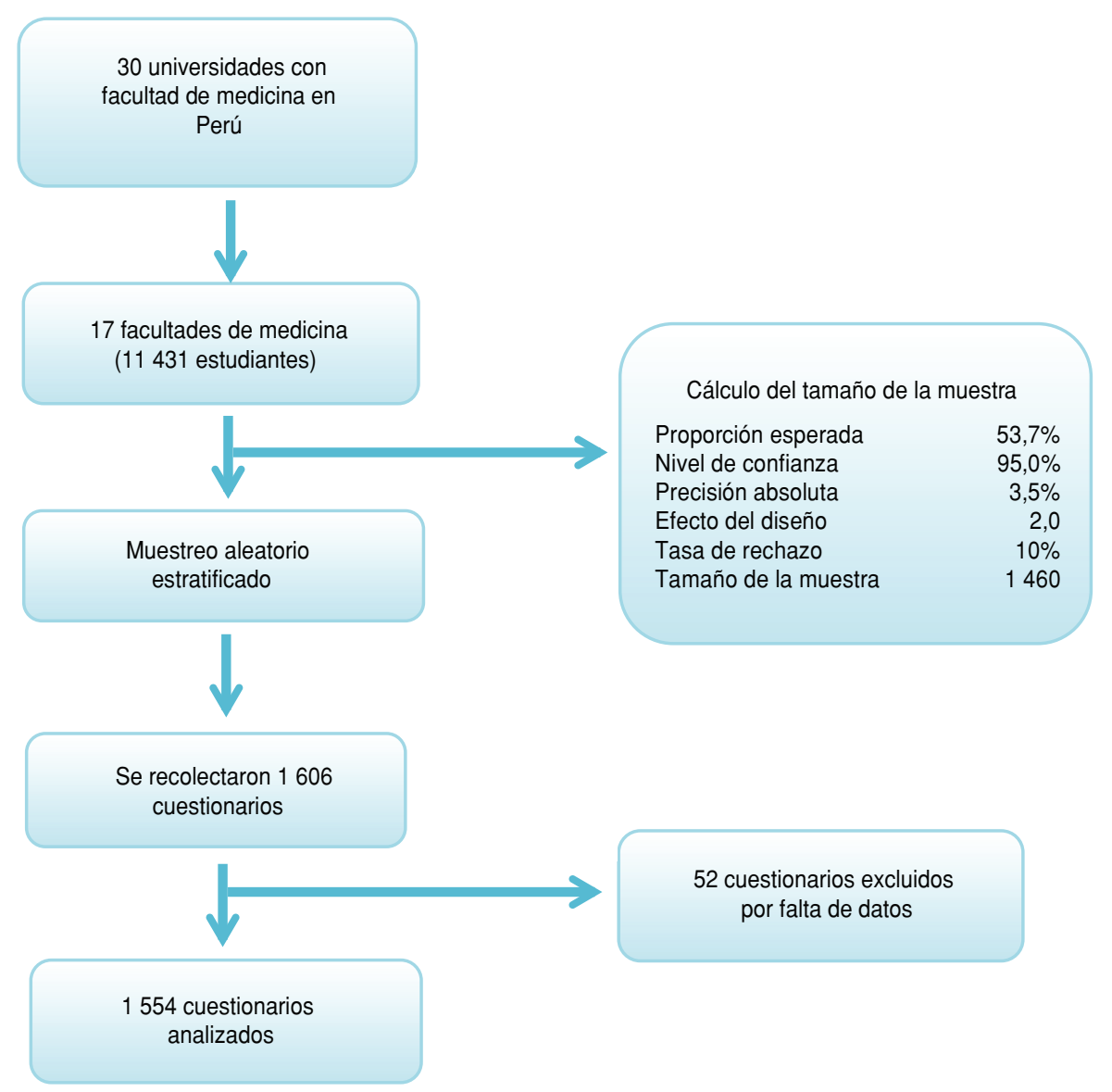

que el cuestionario modificado mantenía excelentes características (coeficiente alfa de Cronbach de 0,82 para los conocimientos y 0,74 para la actitud; correlación intraclase de $83 \%$ y $73 \%$, respectivamente; índice KMO de 0,$88 ; y$ esfericidad $<0,05$ ).

\section{Análisis de los datos}

Se realizó el análisis simple y bifactorial de los resultados para obtener las frecuencias absolutas y relativas para las variables categóricas, y la media, la mediana y la desviación estándar para las variables cuantitativas, mediante el paquete estadístico SPSS versión 17. Por ser una muestra seleccionada por conglomerados en dos etapas, se realizó el análisis con ajuste de la correlación intraclase, mediante el comando soy para análisis de muestras complejas del paquete estadístico STATA 11.0. Se modelaron razones de prevalencia mediante análisis bifactorial y multifactorial con modelos lineales generalizados. Se utilizó un nivel de significación de $P<0,05$.

El cuestionario y la investigación recibieron la aprobación del Comité Institucional de Ética en Investigación de la Universidad San Martín de Porres, de Lima, Perú, (RCEI-18) y se obtuvo la autorización de cada decano o director de las escuelas participantes antes del inicio del trabajo de campo. El cuestionario fue autoaplicable y anónimo, y contaba con una declaración de consentimiento informado; no se destinó un tiempo determinado para su completamiento.

\section{RESULTADOS}

Se aplicaron 1606 encuestas, de las cuales se eliminaron $52(3,2 \%)$ por faltar datos generales o por tener más de $30 \%$ de las preguntas en blanco. De los 1554 encuestados que entraron en el análisis (figura 1), la edad promedio fue de 20,5 $\pm 2,9$ años (de 15 a 44); 51,0\% del total de encuestados eran mujeres.

Los cursos extracurriculares a los que menos estudiantes asistieron fueron los de lectura crítica $(3,3 \%)$ y redacción científica $(4,2 \%)$; además $66,3 \%$ refirió no haber recibido ningún curso extracurricular en temas de investigación científica; $83,7 \%$ no pertenecía a ningún grupo de investigación; 44,5\% tenía dificultades con el uso de programas estadísticos (SPSS, STATA, entre otros) y 59,6\% no había realizado trabajo de investigación alguno (cuadro 2). 
CUADRO 2. Características de las investigaciones desarrolladas por los estudiantes participantes en el estudio, Perú, 2011

\begin{tabular}{|c|c|c|}
\hline Característica & $\begin{array}{l}\text { Número de } \\
\text { estudiantes }\end{array}$ & Porcentaje \\
\hline \multicolumn{3}{|l|}{ Curso extracurricular } \\
\hline Metodología de la investigación científica & 310 & 20,0 \\
\hline Estadística & 97 & 6,2 \\
\hline Redacción científica & 65 & 4,2 \\
\hline Lectura crítica & 51 & 3,3 \\
\hline Ninguno & 1031 & 66,3 \\
\hline \multicolumn{3}{|l|}{ Grupo de investigación } \\
\hline Sí & 254 & 16,3 \\
\hline No & 1300 & 83,7 \\
\hline \multicolumn{3}{|l|}{ Dificultades encontradas para hacer la investigacióna } \\
\hline Selección del diseño & 429 & 27,6 \\
\hline Programas estadísticos & 692 & 44,5 \\
\hline Selección de las pruebas estadísticas & 446 & 28,7 \\
\hline Interpretación del análisis estadístico y los resultados & 371 & 23,9 \\
\hline Redacción del informe final & 251 & 16,2 \\
\hline Búsqueda bibliográfica & 291 & 18,7 \\
\hline Análisis del material bibliográfico & 206 & 13,3 \\
\hline Prueba de hipótesis & 290 & 18,7 \\
\hline Selección de la muestra y el muestreo & 326 & 21,0 \\
\hline Planteamiento de la pregunta de investigación & 341 & 21,9 \\
\hline \multicolumn{3}{|l|}{ Participación en investigaciones } \\
\hline Sí, actualmente & 358 & 23,0 \\
\hline Sí, anteriormente & 436 & 28,1 \\
\hline Nunca ha participado & 760 & 48,9 \\
\hline \multicolumn{3}{|l|}{ Número de trabajos de investigación ${ }^{b}$} \\
\hline Ninguno & 926 & 59,6 \\
\hline 1 ó 2 & 546 & 35,1 \\
\hline$>2$ & 82 & 5,3 \\
\hline \multicolumn{3}{|l|}{ Número de proyectos científicos ${ }^{b}$} \\
\hline Ninguno & 573 & 36,9 \\
\hline 1 ó 2 & 728 & 46,8 \\
\hline$>2$ & 253 & 16,3 \\
\hline
\end{tabular}

a Los participantes podían responder más de una opción.

b Se consideraron todos los trabajos y proyectos en los que ha participado durante la carrera.

Según el análisis bifactorial, las variables independientes que mostraron asociación significativa con el nivel de conocimientos y la actitud hacia la investigación científica fueron el sexo, la edad, haber realizado cursos extracurriculares, pertenecer a un grupo de investigación, el tipo de universidad, el año de estudio, la participación en investigación y la cantidad de proyectos y trabajos realizados (cuadro 3). Estas variables se utilizaron en el análisis multifactorial.

Con respecto al nivel de conocimientos y la actitud, se encontró que solamente $46,7 \%$ de los encuestados tenía un buen conocimiento sobre la investigación y 37,7\% una adecuada actitud hacia ella (cuadro 4).

En el análisis multifactorial, las variables asociadas con el buen nivel de conocimientos fueron: estar en un año académico mayor del 3. ${ }^{\circ}$ (el nivel de conocimientos aumentó al avanzar en la carrera, desde $66 \%$ en $4 .^{\circ}$ año y $58 \%$ en $5 .^{\circ}$ hasta $72 \%$ en $6 .^{\circ}$ año), pertenecer a un grupo de investigación (aumentó en $69 \%$ las probabilidades de tener un buen nivel de conocimientos); así como contar con un asesor (elevó en 31\% esa probabilidad). Las variables edad, participar en más de dos proyectos y tener una revista en su facultad perdieron su significación estadística al realizar el ajuste multifactorial (cuadro 5). Por su parte, estudiar en una universidad con presupuesto para la actividad investigativa no aumentó la probabilidad de tener un buen nivel de conocimientos sobre investigación (cuadro 5).

La actitud hacia la investigación estuvo asociada con el sexo (las mujeres tuvieron $17 \%$ mayor probabilidad de tener una actitud adecuada que los varones); llama la atención que los estudiantes que pertenecían a un grupo de investigación, participaban en más de dos proyectos y estudiaban en una universidad con presupuesto para investigación tuvieron $40 \%, 16 \%$ y $29 \%$ menos probabilidad de tener una actitud adecuada hacia la investigación. Otro resultado de interés fue que tener un asesor aumentó en $40 \%$ la posibilidad de tener una adecuada actitud hacia la investigación (cuadro 6).

En cuanto a las diferentes universidades, se encontraron fuertes variaciones en el buen nivel de conocimientos de sus alumnos (de $19,2 \%$ a $100 \%$ ); contradictoriamente, algunas de estas universidades presentaron altos porcentajes de alumnos con inadecuada actitud, y viceversa (cuadro 7). En $11(64,7 \%)$ de las 17 universidades, más de $50 \%$ de los alumnos mostró un buen conocimiento; no obstante, más de $50 \%$ de los alumnos tenía una inadecuada actitud en 12 $(70,6 \%)$ de ellas.

\section{DISCUSIÓN}

El nivel de conocimientos y la actitud en la población estudiada muestra una disminución de 7,0 y 34,2 puntos porcentuales, respectivamente, con relación a los resultados obtenidos en una investigación similar realizada en 2005 (12), que abarcó a la mayoría de las facultades de medicina participantes en el presente estudio. Esto podría estar relacionado con diversos factores, como la disminución de la producción científica en la etapa de pregrado (13) y la tendencia a formar profesionales dedicados predominantemente a actividades asistenciales. Adicionalmente, en 1991 se modificó la Ley Universitaria $N^{\circ} 23733$ mediante el Decreto Legislativo 739 (14) que, entre otras medidas, estableció que se puede obtener el título profesional mediante las modalidades que crea conveniente la universidad, además de la presentación de una tesis, lo que ha reducido el número de estudiantes que realizan investigaciones para titularse (15).

Se comprobó que las probabilidades de tener un buen conocimiento sobre la investigación aumentaron a partir del 4. ${ }^{\circ}$ año de estudios (cuadro 5). Esto se podría explicar por la asistencia a cursos más inclinados hacia la investigación, como epidemiología, y la participación en proyectos o talleres de investigación, además de la incorporación al plan de tesis, todo ello programado en los últimos años de la carrera. No obstante, estos cursos suman menos de $8 \%$ del currícu- 
CUADRO 3. Características generales y relacionadas con la investigación de los estudiantes participantes en el estudio, según el nivel de conocimientos y la actitud hacia la investigación, Perú, 2011

\begin{tabular}{|c|c|c|c|c|c|c|c|c|c|c|}
\hline \multirow[b]{3}{*}{ Característica } & \multicolumn{4}{|c|}{ Conocimientos } & \multirow[b]{3}{*}{$P^{a}$} & \multicolumn{4}{|c|}{ Actitud } & \multirow[b]{3}{*}{$P^{a}$} \\
\hline & \multicolumn{2}{|c|}{ Buenos } & \multicolumn{2}{|c|}{ Insuficientes } & & \multicolumn{2}{|c|}{ Adecuada } & \multicolumn{2}{|c|}{ Inadecuada } & \\
\hline & No. & $\%$ & No. & $\%$ & & No. & $\%$ & No. & $\%$ & \\
\hline Hombres & 356 & 22,9 & 405 & 26,1 & \multirow{2}{*}{$>0,05$} & 328 & 21,1 & 433 & 27,9 & \multirow{2}{*}{$<0,05$} \\
\hline Mujeres & 370 & 23,8 & 423 & 27,2 & & 258 & 16,6 & 535 & 34,4 & \\
\hline \multicolumn{11}{|l|}{ Edad (años) } \\
\hline \multicolumn{11}{|l|}{ Año de estudio } \\
\hline $1 .^{\circ}$ & 129 & 8,3 & 268 & 17,2 & $<0,05$ & 134 & 8,6 & 263 & 16,9 & $>0,05$ \\
\hline $2 .^{\circ}$ & 150 & 9,7 & 178 & 11,5 & $>0,05$ & 128 & 8,2 & 200 & 12,9 & $>0,05$ \\
\hline $3 .{ }^{\circ}$ & 134 & 8,6 & 148 & 9,5 & $>0,05$ & 121 & 7,8 & 161 & 10,4 & $<0,05$ \\
\hline $4 .^{\circ}$ & 113 & 7,3 & 90 & 5,8 & $>0,05$ & 78 & 5,0 & 125 & 8,0 & $>0,05$ \\
\hline $5 .^{\circ}$ & 100 & 6,4 & 88 & 5,7 & $<0,05$ & 68 & 4,4 & 120 & 7,7 & $>0,05$ \\
\hline $6 .^{\circ}$ & 100 & 6,4 & 56 & 3,6 & $<0,05$ & 57 & 3,7 & 99 & 6,4 & $>0,05$ \\
\hline Lectura crítica & 25 & 1,6 & 26 & 1,7 & & 21 & 1,4 & 30 & 1,9 & \\
\hline Ninguno & 444 & 28,6 & 614 & 39,5 & & 345 & 22,2 & 713 & 45,9 & \\
\hline Grupo de investigación & & & & & & & & & & \\
\hline Sí pertenece & 190 & 12,2 & 64 & 4,1 & & 155 & 10,0 & 99 & 6,4 & \\
\hline No pertenece & 536 & 34,5 & 764 & 49,2 & $<0,05$ & 431 & 27,7 & 869 & 55,9 & $<0,05$ \\
\hline Participación en proyectos o trabajos de investigac & & & & & & & & & & \\
\hline Actualmente & 229 & 14,7 & 129 & 8,3 & & 189 & 12,2 & 169 & 10,9 & \\
\hline Anteriormente & 249 & 16,0 & 187 & 12,0 & $<0,05$ & 186 & 12,0 & 250 & 16,1 & $<0,05$ \\
\hline Dificultades para realizar la investigación & & & & & & & & & & \\
\hline Ninguna & 74 & 4,8 & 22 & 1,4 & & 34 & 2,2 & 62 & 3,4 & \\
\hline Al menos una dificultad & 652 & 42,0 & 806 & 51,9 & & 552 & 35,5 & 906 & 58,3 & \\
\hline Selección del diseño & 202 & 13,0 & 227 & 14,6 & & 143 & 9,2 & 286 & 18,4 & \\
\hline Planteamiento de la pregunta de investigación & 141 & 9,1 & 200 & 12,9 & & 112 & 7,2 & 229 & 14,7 & \\
\hline Tipo de universidad & & & & & & & & & & \\
\hline Privada & 449 & 28,9 & 698 & 44,9 & & 423 & 27,2 & 724 & 46,6 & \\
\hline Pública & 277 & 17,8 & 130 & 8,4 & $<0,05$ & 163 & 10,5 & 244 & 15,7 & $>0,05$ \\
\hline Número de trabajos & & & & & & & & & & \\
\hline Ninguno & 408 & 26,3 & 518 & 33,3 & & 319 & 20,5 & 607 & 39,1 & \\
\hline 1 ó 2 & 270 & 17,4 & 276 & 17,8 & $<0,05$ & 223 & 14,4 & 323 & 20,8 & $<0,05$ \\
\hline$>2$ & 48 & 3,1 & 34 & 2,2 & & 44 & 2,8 & 38 & 2,5 & \\
\hline Número de proyectos & & & & & & & & & & \\
\hline Ninguno & 207 & 13,3 & 366 & 23,5 & & 179 & 11,5 & 394 & 25,3 & \\
\hline 1 ó 2 & 365 & 23,5 & 363 & 23,4 & $<0,05$ & 281 & 18,1 & 447 & 28,8 & $<0,05$ \\
\hline$>2$ & 154 & 9,9 & 99 & 6,4 & $<0,05$ & 126 & 8,1 & 127 & 8,2 & $<0,05$ \\
\hline
\end{tabular}

a Según la prueba de la ji al cuadrado; nivel de significación $P<0,05$.

lum total de las facultades de medicina comprendidas en este estudio.

El hecho de que pertenecer a un grupo de investigación aumentó en $69 \%$ la probabilidad de tener un buen nivel de conocimientos sobre la investigación abogaría a favor de mantener vigente estos espacios, que posibilitan el desarrollo continuo de los estudiantes en el campo científico al permitirles relacionarse con otros estudiantes y profesionales investigadores. Los estudiantes interesados que se agrupan principalmente en asociaciones científicas podrían profundizar estos conocimientos en investigación mediante cursos, talleres, o jornadas y congresos científicos, ya que todas estas actividades fomentan la investigación 
CUADRO 4. Nivel de conocimientos y actitud relacionados con la investigación de los estudiantes participantes en el estudio, Perú, 2011

\begin{tabular}{|c|c|c|c|}
\hline Variable & No. & $\%$ & IC $95 \%{ }^{a}$ \\
\hline \multicolumn{4}{|c|}{ Nivel de conocimientos } \\
\hline Bueno & 726 & 46,7 & $34,5-59,4$ \\
\hline Insuficiente & 828 & 53,3 & $40,6-65,5$ \\
\hline \multicolumn{4}{|l|}{ Actitud } \\
\hline Adecuada & 586 & 37,7 & $31,5-44,3$ \\
\hline Inadecuada & 968 & 62,3 & $55,7-68,5$ \\
\hline
\end{tabular}

y las publicaciones científicas desde el pregrado (16).

También, la asignación de un presupuesto específico para la realización de actividades científicas y el desarrollo y la programación de trabajos de investigación deben ser factores asociados con el buen nivel de conocimientos sobre la investigación, como ya se ha constatado en otros estudios (16). Sin embargo, en este estudio se encontró que contar con un presupuesto para la investigación redujo la probabilidad de tener un buen nivel de conocimientos y una adecuada actitud respecto a la investigación, lo que podría deberse a deficiencias en la ejecución del presupuesto dedicado a la investigación en las universidades estudiadas que lo tenían, algo que aún debe investigarse.

Aunque es de esperar que los estudiantes con mejor actitud buscarían participar en más proyectos por iniciativa propia $(13,17)$, llama la atención que haber realizado más de dos proyectos o trabajos de investigación no se asoció con un buen conocimiento sobre la investigación ni una adecuada actitud hacia ella en la muestra estudiada. Esto podría reflejar la existencia de deficiencias en el mismo proceso de investigación realizado por los alumnos.

Las mujeres tuvieron $17 \%$ más probabilidades de presentar una adecuada ac-

CUADRO 5. Análisis de los factores asociados con el nivel de conocimientos sobre investigación de los estudiantes participantes en el estudio, Perú, 2011

\begin{tabular}{|c|c|c|c|c|c|c|}
\hline \multirow[b]{2}{*}{ Variable } & \multicolumn{3}{|c|}{ Análisis bifactorial ${ }^{a}$} & \multicolumn{3}{|c|}{ Análisis multifactoriala } \\
\hline & $\mathrm{RP}^{\mathrm{b}}$ & IC95\% $\%^{\mathrm{C}}$ & $P^{\mathrm{d}}$ & $\mathrm{RPb}$ & IC95\% ${ }^{c}$ & $P^{\mathrm{d}}$ \\
\hline Edad & 1,05 & $1,00-1,10$ & $<0,05$ & 0,99 & $0,95-1,04$ & $>0,05$ \\
\hline \multicolumn{7}{|l|}{ Sexo } \\
\hline Hombres & 1,00 & & & 1,00 & & \\
\hline Mujeres & 0,99 & $0,87-1,14$ & $>0,05$ & 1,05 & $0,94-1,19$ & $>0,05$ \\
\hline \multicolumn{7}{|l|}{ Año de estudio } \\
\hline $1 .^{\circ}$ & 1,00 & & & 1,00 & & \\
\hline $2 .{ }^{\circ}$ & 1,41 & $0,90-2,20$ & $>0,05$ & 1,31 & $0,90-1,92$ & $>0,05$ \\
\hline $3 .{ }^{\circ}$ & 1,46 & $1,01-2,12$ & $<0,05$ & 1,36 & $0,93-1,98$ & $>0,05$ \\
\hline $4 .^{\circ}$ & 1,71 & $1,13-2,60$ & $<0,05$ & 1,66 & $1,12-2,46$ & $<0,05$ \\
\hline $5 . .^{\circ}$ & 1,64 & $1,93-2,50$ & $<0,05$ & 1,58 & $1,31-2,21$ & $<0,05$ \\
\hline $6 .^{\circ}$ & 1,97 & $1,22-3,17$ & $<0,05$ & 1,72 & $1,01-2,70$ & $<0,05$ \\
\hline \multicolumn{7}{|c|}{ Grupo de investigación } \\
\hline No pertenece & 1,00 & & & 1,00 & & \\
\hline Pertenece & 1,81 & $1,38-2,39$ & $<0,001$ & 1,69 & $1,36-2,01$ & $<0,001$ \\
\hline \multicolumn{7}{|c|}{ Número de proyectos } \\
\hline$\leq 2$ & 1,00 & & & 1,00 & & \\
\hline$>2$ & 1,38 & $1,13-1,70$ & $<0,05$ & 1,10 & $0,88-1,36$ & $>0,05$ \\
\hline \multicolumn{7}{|c|}{ Número de trabajos de investigación } \\
\hline$\leq 2$ & 1,00 & & & 1,00 & & \\
\hline$>2$ & 1,27 & $0,93-1,74$ & $>0,05$ & 1,14 & $0,88-1,49$ & $>0,05$ \\
\hline \multicolumn{7}{|l|}{ Asesores } \\
\hline No tiene & 1,00 & & & 1,00 & & \\
\hline Tiene & 0,57 & $0,40-0,83$ & $<0,05$ & 1,31 & $1,11-1,54$ & $<0,05$ \\
\hline \multicolumn{7}{|c|}{ Revista en la facultad } \\
\hline No tiene & 1,00 & & & 1,00 & & \\
\hline Tiene & 0,60 & $0,41-0,90$ & $<0,05$ & 0,76 & $0,54-1,06$ & $>0,05$ \\
\hline \multicolumn{7}{|c|}{ Presupuesto para investigación } \\
\hline No tiene & 1,00 & & & 1,00 & & \\
\hline Tiene & 0,55 & $0,39-0,77$ & $<0,05$ & 0,51 & $0,37-0,71$ & $<0,001$ \\
\hline
\end{tabular}

a Análisis ajustado para el muestreo en dos etapas mediante el comando svy para análisis de muestras complejas de paquete estadístico STATA 11.0 .

b RP: razón de prevalencia ajustada con modelos lineales generalizados.

c IC95\%: intervalo de confianza de 95\% corregido mediante el comando svy para análisis de muestras complejas del paquete estadístico STATA 11.0

d Regresión logística logarítmica de Poisson; nivel de significación $P<0,05$

titud hacia la investigación, un hallazgo singular de este estudio, que podría reflejar una mayor predisposición de las estudiantes hacia la investigación, en comparación con sus compañeros varones.

Contar con un asesor se asoció con tener una actitud adecuada y buenos conocimientos, sin dudas debido a la importancia de la labor de los mentores científicos (18-20) que, además de ayudar en la concepción y guiar el trabajo, forman al estudiante en aspectos éticos y evitan conductas inadecuadas que por desconocimiento puedan considerarse normales. El incremento de 31\% en la probabilidad de tener buen conocimiento sobre la investigación cuando se cuenta con un asesor debidamente capacitado coincide con lo expresado por Gutiérrez y Mayta, quienes puntualizan que esta es una condición necesaria para obtener buenos resultados en investigación (21).

Una estrategia clave para la formación de investigadores, apoyada por los resultados encontrados, sería reforzar el trabajo con los grupos de investigación. Este tipo de espacios genera entornos que favorecen y promueven la interacción asesor (investigador)-alumno. Cabe resaltar que $75 \%$ de las publicaciones científicas estudiantiles generadas en Perú son de autoría conjunta con profesionales, lo que demuestra la eficacia de la participación de estudiantes en la investigación cuando están asesorados por investigadores con experiencia (11, 13, 20, 22, 23).

El alto porcentaje de alumnos con insuficientes conocimientos sobre investigación e inadecuada actitud hacia ella podría reflejar una mala relación entre lo que se enseña y lo que se exige al alumno en cuanto a la investigación; esto se manifestaría, a su vez, en el bajo número de publicaciones estudiantiles en revistas de mayor visibilidad internacional.

Aunque el diseño del presente estudio es transversal analítico y no permite establecer relaciones de causalidad entre las asociaciones encontradas, estos resultados pueden ser útiles para diseñar mejores estrategias y sirve de punto de partida para estudios posteriores más completos, basados en muestras más representativas de toda la población estudiantil de pregrado de medicina en Perú.

No obstante esas limitaciones, se puede concluir que el nivel de conocimientos en investigación hallado en el presente estudio es, en general, deficiente. Los factores 
CUADRO 6. Análisis de los factores asociados con la actitud hacia la investigación de los estudiantes participantes en el estudio, Perú, 2011

\begin{tabular}{|c|c|c|c|c|c|c|}
\hline \multirow[b]{2}{*}{ Variables } & \multicolumn{3}{|c|}{ Análisis bifactoriala $^{a}$} & \multicolumn{3}{|c|}{ Análisis multifactoriala } \\
\hline & $\mathrm{RP}^{\mathrm{b}}$ & IC95\% ${ }^{\mathrm{C}}$ & $P^{\mathrm{d}}$ & $\mathrm{RP}^{\mathrm{b}}$ & IC95\% ${ }^{\mathrm{C}}$ & $P^{d}$ \\
\hline Edad & 0,99 & $0,97-1,02$ & $>0,05$ & 0,99 & $0,97-1,02$ & $>0,05$ \\
\hline \multicolumn{7}{|l|}{ Sexo } \\
\hline Hombres & 1,00 & & & 1,00 & & \\
\hline Mujeres & 1,19 & $1,10-1,28$ & $<0,001$ & 1,17 & $1,07-1,26$ & $<0,001$ \\
\hline \multicolumn{7}{|l|}{ Año de estudio } \\
\hline $1 .^{\circ}$ & 1,00 & & \multirow{6}{*}{$>0,05$} & 1,00 & & \multirow{6}{*}{$>0,05$} \\
\hline $2 .{ }^{\circ}$ & 0,92 & $0,73-1,15$ & & 0,94 & $0,76-1,15$ & \\
\hline $3 .{ }^{\circ}$ & 0,86 & $0,70-1,06$ & & 0,92 & $0,75-1,11$ & \\
\hline $4 .^{\circ}$ & 0,93 & $0,79-1,09$ & & 0,99 & $0,84-1,18$ & \\
\hline $5 .^{\circ}$ & 0,69 & $0,75-1,23$ & & 1,04 & $0,83-1,32$ & \\
\hline $6 .^{\circ}$ & 0,96 & $0,70-1,31$ & & 1,11 & $0,78-1,58$ & \\
\hline \multicolumn{7}{|c|}{ Grupo de investigación } \\
\hline No pertenece & 1,00 & & & 1,00 & & \\
\hline Pertenece & 0,58 & $0,50-0,68$ & $<0,001$ & 0,60 & $0,53-0,69$ & $<0,001$ \\
\hline \multicolumn{7}{|c|}{ Número de proyectos } \\
\hline$\leq 2$ & 1,00 & & & 1,00 & & \\
\hline$>2$ & 0,78 & $0,63-0,96$ & $<0,05$ & 0,84 & $0,71-0,99$ & $<0,05$ \\
\hline \multicolumn{7}{|c|}{ Número de trabajos de investigación } \\
\hline$\leq 2$ & 1,00 & & & 1,00 & & \\
\hline$>2$ & 0,73 & $0,50-1,07$ & $>0,05$ & 0,88 & $0,67-1,16$ & $>0,05$ \\
\hline \multicolumn{7}{|l|}{ Asesores } \\
\hline No tiene & 1,00 & & & 1,00 & & \\
\hline Tiene & 1,02 & $0,80-1,31$ & $>0,05$ & 1,40 & $1,07-1,84$ & $<0,05$ \\
\hline \multicolumn{7}{|c|}{ Revista en la facultad } \\
\hline No tiene & 1,00 & & & 1,00 & & \\
\hline Tiene & 1,04 & $0,85-1,28$ & $>0,05$ & 1,03 & $0,82-1,30$ & $>0,05$ \\
\hline \multicolumn{7}{|c|}{ Presupuesto para investigación } \\
\hline No tiene & 1,00 & & & 1,00 & & \\
\hline Tiene & 0,96 & $0,75-1,23$ & $>0,05$ & 0,71 & $0,58-0,86$ & $<0,05$ \\
\hline
\end{tabular}

a Análisis ajustado para el muestreo en dos etapas mediante el comando svy para análisis de muestras complejas del paquete estadístico STATA 11.0

b RP: razón de prevalencia ajustada con modelos lineales generalizados.

c IC95\%: intervalo de confianza de 95\% corregido mediante el comando svy para análisis de muestras complejas del paquete estadístico STATA 11.0.

d Regresión logística logarítmica de Poisson; nivel de significación $P<0,05$. asociados con los buenos conocimientos sobre investigación fueron: estar en los últimos tres años de la carrera, pertenecer a un grupo de investigación y contar con asesores. La actitud ante la investigación fue también, en general, inadecuada. Los factores asociados con una buena actitud fueron: ser mujer, pertenecer a un grupo de investigación, participar en más de dos proyectos de investigación y contar con asesores.

Los resultados presentados demuestran que es prioritario desarrollar estrategias que garanticen mejoras sustanciales en la producción científica desde el pregrado. Para transformar la situación negativa encontrada, se deben poner en marcha estrategias que abarquen desde ajustes en el contenido curricular - como la inclusión de una secuencia lógica y coherente de asignaturas que desarrolle en los estudiantes las competencias necesarias para llevar a cabo investigaciones científicas de calidad al finalizar la carrera- hasta medidas que impidan la adopción de iniciativas y regulaciones contrarias al desarrollo de las investigaciones en el pregrado.

Agradecimientos. Los miembros del Comité Permanente Científico de la SOCIMEP que participaron en la recolección de datos son: Cristian Abia Meashima, Guillermo Acuña Castañeda, Johana Ávila Figueroa, Carlos Chunga Flores, Franklin Correa Prieto, Henry Delgado Castillo, Carla Espinoza Neyra, Jersson Mamani, Álvaro Taype Rondán, Javier Torres Va-

CUADRO 7. Nivel de conocimientos sobre investigación y actitud ante ella, según la universidad de procedencia de los estudiantes participantes en el estudio, Perú, 2011

\begin{tabular}{|c|c|c|c|c|c|c|c|c|c|c|}
\hline \multirow[b]{3}{*}{ Universidad y tipo } & \multicolumn{5}{|c|}{ Nivel de conocimientos } & \multicolumn{5}{|c|}{ Actitud } \\
\hline & \multicolumn{2}{|c|}{ Bueno } & \multicolumn{2}{|c|}{ Insuficiente } & \multirow{2}{*}{$\begin{array}{c}\text { Desviación } \\
\text { estándar }\end{array}$} & \multicolumn{2}{|c|}{ Adecuada } & \multicolumn{2}{|c|}{ Inadecuada } & \multirow{2}{*}{$\begin{array}{l}\text { Desviación } \\
\text { estándar }\end{array}$} \\
\hline & No. & $\%$ & No. & $\%$ & & No. & $\%$ & No. & $\%$ & \\
\hline \multicolumn{11}{|l|}{ Pública } \\
\hline Universidad Nacional del Altiplano & 41 & 82,0 & 9 & 18,0 & 1,25 & 23 & 46,0 & 27 & 54,0 & 5,19 \\
\hline Universidad Nacional de la Amazonía Peruana & 20 & 57,1 & 15 & 42,9 & 1,98 & 10 & 28,6 & 25 & 71,4 & 8,69 \\
\hline Universidad Nacional de Cajamarca & 36 & 76,6 & 11 & 23,4 & 1,52 & 10 & 21,3 & 37 & 78,7 & 5,67 \\
\hline Universidad Nacional de Ucayali & 15 & 39,5 & 23 & 60,5 & 1,62 & 20 & 52,6 & 18 & 47,4 & 7,97 \\
\hline Universidad Nacional José Faustino Sánchez Carrión & 27 & 62,8 & 16 & 37,2 & 1,66 & 14 & 32,6 & 29 & 67,4 & 6,39 \\
\hline Universidad Nacional Pedro Ruiz Gallo & 26 & 76,5 & 8 & 23,5 & 1,62 & 15 & 44,1 & 19 & 55,9 & 7,79 \\
\hline Universidad Nacional San Antonio Abad del Cusco & 43 & 74,1 & 15 & 25,9 & 1,87 & 30 & 51,7 & 28 & 48,3 & 6,53 \\
\hline Universidad Nacional San Luis Gonzaga & 41 & 58,6 & 29 & 41,4 & 1,72 & 37 & 52,9 & 33 & 47,1 & 6,06 \\
\hline Universidad Nacional de Piura & 28 & 87,5 & 4 & 12,5 & 1,31 & 4 & 12,5 & 28 & 87,5 & 7,22 \\
\hline \multicolumn{11}{|l|}{ Privada } \\
\hline Universidad Católica Santa María & 69 & 56,1 & 54 & 43,9 & 1,95 & 37 & 30,1 & 86 & 69,9 & 7,05 \\
\hline Universidad Cesar Vallejo & 8 & 38,1 & 13 & 61,9 & 2,18 & 12 & 57,1 & 9 & 42,9 & 9,47 \\
\hline Universidad de Chiclayo & 54 & 100,0 & 0 & 0 & 0,63 & 22 & 40,7 & 32 & 59,3 & 10,92 \\
\hline Universidad Privada de Tacna & 37 & 74,0 & 13 & 26,0 & 1,55 & 16 & 32,0 & 34 & 68,0 & 8,76 \\
\hline Universidad Privada Antenor Orrego & 105 & 33,0 & 213 & 67,0 & 2,46 & 144 & 45,3 & 174 & 54,7 & 9,69 \\
\hline Universidad Privada de Ciencias Aplicadas & 27 & 34,2 & 52 & 65,8 & 2,22 & 42 & 53,2 & 37 & 46,8 & 9,50 \\
\hline Universidad Ricardo Palma & 37 & 19,2 & 156 & 80,8 & 2,10 & 60 & 31,1 & 133 & 68,9 & 10,28 \\
\hline Universidad Privada San Martin de Porres & 112 & 36,2 & 197 & 63,8 & 2,24 & 90 & 29,1 & 219 & 70,9 & 8,26 \\
\hline Total & 726 & 46,7 & 828 & 53,3 & 2,33 & 586 & 37,7 & 968 & 62,3 & 8,88 \\
\hline
\end{tabular}


lencia, Gelsing Vásquez García, Eduardo Vélez Segovia y Enrique Zumaeta Saavedra. Una versión de este trabajo se presentó en el XXV Congreso Científico
Nacional de Estudiantes de Medicina, de la Sociedad Científica Médico Estudiantil Peruana (SOCIMEP), celebrado en Cusco, Perú, del 2 al 6 de agosto de 2011.

\section{REFERENCIAS}

1. Narváez JG, Sánchez JJ, Velásquez AT. La investigación como factor estratégico de desarrollo en Colombia: investigar o caer en la marginalidad planetaria. Rev Esc Administracion Negocios. 2001;42-43:18-34.

2. Cabezas C. Necesidad de la investigación en salud para contribuir a la equidad, la salud y el desarrollo. Rev Peru Med Exp Salud Publica. 2010;27(3):310-11.

3. Caballero P, Yagui M, Espinoza M, Castilla T, Granados A, Velásquez A, et al. Prioridades regionales y nacionales de investigación en salud, Perú 2010-2014: un proceso con enfoque participativo y descentralista. Rev Peru Med Exp Salud Publica. 2010;27(3):367-72.

4. Albornoz M. El estado de la ciencia 2009. Principales indicadores de ciencia y tecnología iberoamericanos/interamericanos. Informe del Centro de Estudios sobre Ciencia, Desarrollo y Educación Superior. Buenos Aires: Red Iberoamericana de Indicadores de Ciencia y Tecnología; 2009. Disponible en: http://www.revistacts.net/files/Portafolio/ El_estado_de_la_ciencia_2009.pdf Acceso el 30 de noviembre de 2012

5. Huamaní C, Mayta-Tristán P. Producción científica peruana en medicina y redes de colaboración: análisis del Science Citation Index 2000-2009. Rev Peru Med Exp Salud Publica. 2010;27(3):315-25.

6. D'Eon M, Harris C. If students are not customers, what are they? Acad Med. 2000;75(12): 1173-77.
7. Aslam F, Shakir M, Qayyum MA. Why medical students are crucial to the future of research in South Asia? PLoS Med. 2005;2(11):1110-1.

8. Pedraza V. Las facultades de medicina y la formación de los médicos. Educ Med. 1999;2:53-60.

9. Franco C, Rodríguez-Morales AJ. Revistas científicas de estudiantes de medicina. Gac Med Caracas. 2009;117(1):70-90.

10. Huamaní C, Mayta P, Rodríguez A. Publicar desde pregrado. Interciencia. 2008;33(11):785.

11. Khan H, RizwanulHaq M, Waheed A, Ameen $\mathrm{M}$, Fatmi Z. Knowledge and attitudes about health research amongst a group of Pakistani medical students. BMC Med Educ. 2006;6:54-61.

12. Díaz-Vélez C, Manrique L, Galán E, Apolaya M. Conocimientos, actitudes y prácticas en investigación de los estudiantes de pregrado de facultades de medicina en el Perú. Acta Med Peru. 2008;25(1):9-15.

13. Ángel $\mathrm{A}$, Botero $\mathrm{H}$, González $\mathrm{D}$, Ospina L, Velasco M, Ocampo M. Interés de los estudianvest Med Estudiantil Latinoam. 2010;15(1):9-13.

14. Galán-Rodas E, Díaz-Vélez C, Mejía C. Intención en la modalidad de titulación de internos de medicina en el Perú, 2010. Rev Peru Med Exp Salud Publica. 2011;28(2):390-9.

15. Góngora Prado M. El Estado, la universidad peruana y el drama de la investigación científica. Escritura Pensamiento. 2002;5(11):43-57.

16. Molina-Ordóñez J, Huamaní C, MaytaTristán P. Apreciación estudiantil sobre la capacitación universitaria en investigación: tes de medicina por la investigación. Cien In-
Conflicto de interés. Los autores pertenecen a la Sociedad Científica Médico Estudiantil Peruana. estudio preliminar. Rev Peru Med Exp Salud Publica. 2008;25(3):325-9.

17. Alarcón-Villaverde J, Romaní F, Gutiérrez C. Publicaciones científicas estudiantiles producidas en el curso de epidemiología de la Facultad de Medicina de la Universidad Nacional Mayor de San Marcos durante el período 2003-2009. An Fac Med. 2010;71(2):111-6.

18. Valenzuela G. Irregularidades en la publicación de trabajos científicos. An Fac Med. 2008;69(1):56-8.

19. Huamaní C, Mayta P, Rodríguez A. Irregularidades éticas en la investigación estudiantil. An Fac Med. 2008;69(2):146.

20. Huamaní C, Chávez-Solís P, Mayta-Tristán P. Aporte estudiantil en la publicación de artículos científicos en revistas médicas indizadas en Scielo-Perú, 1997-2005. An Fac Med. 2008;69(1):42-5.

21. Gutiérrez C, Mayta P. Publicación desde el pregrado en Latinoamérica: importancia, limitaciones y alternativas de solución. CIMEL. 2003;8(1):54-60.

22. Salinas J, Vildózola H. Investigación en pregrado: dificultades y posibles soluciones. An Fac Med. 2008;69(3):216

23. Reyes L, Aular J, Palencia J, Muñoz D. Una visión integradora de la investigación estudiantil en pregrado. Rev Cien Soc. 2010;16 (2):250-9.

Manuscrito recibido el 28 de marzo de 2012. Aceptado para publicación, tras revisión, el 19 de septiembre de 2012.
ABSTRACT

\section{Factors associated with level of knowledge and attitudes toward research among medical students in Peru, 2011}

Objective. To identify the factors associated with level of knowledge and attitudes toward research among undergraduate medical students in Peru.

Methods. In this analytical cross-sectional study, a validated questionnaire on knowledge, attitudes, and practices related to research was applied to a representative sample of students at 17 Peruvian medical schools. The categorical and quantitative variables were subjected to simple two-factor analysis with adjustment for intraclass correlation. Prevalence ratios were derived on the basis of generalized linear models using bifactorial and multifactorial analysis.

Results. Questionnaires from 1554 students (51.0\% women) with an average age of $20.5 \pm 2.86$ years were analyzed. Only $46.7 \%$ of the respondents had a good level of knowledge and $37.7 \%$ had appropriate attitudes toward research. The variables that correlated with a good level of knowledge were the respondent having completed at least four years of academic study, belonging to a research group, and having an adviser. Having appropriate attitudes toward research correlated with being a woman and having an adviser. No differences were found between the level of knowledge and attitudes of the students at the universities studied.

Conclusions. In general, the level of knowledge about research was unsatisfactory and the attitudes toward it were inappropriate. In order to change this situation, it will be critical to make changes in the curriculum to ensure that students engage in research from the beginning of their academic careers and develop research skills in their final years of study.

Key words Education, medical, undergraduate; health research policy; research promotion; Peru. 